\title{
Design and Analysis of Thinned Array Pattern Reconfigurable Antenna to Enlarge the Scanning Range
}

\author{
Zhangjing Wang, Xing Fan, Haijuan Cui, and Shaoqiu Xiao \\ School of Electronic Engineering, University of Electronic Science and Technology of China, Chengdu 611731, China \\ Correspondence should be addressed to Zhangjing Wang; 1310466336@qq.com
}

Received 24 December 2015; Revised 13 February 2016; Accepted 18 February 2016

Academic Editor: Ahmed A. Kishk

Copyright ( 2016 Zhangjing Wang et al. This is an open access article distributed under the Creative Commons Attribution License, which permits unrestricted use, distribution, and reproduction in any medium, provided the original work is properly cited.

\begin{abstract}
A novel thinned array with symmetric distribution along the array center is proposed in this paper. The proposed symmetric thinned array is based on the theory of unequally spaced array and the amplitude of each element in the array can be changed by introducing the weighted function. The pattern of the proposed array can be properly adjusted by changing the weighted function and the amplitude of the weighted factor, which obviously releases new degrees of freedom in array design. It has advantages such as low side lobe level (SLL) in the visible region, no grating lobes, and low nearby side lobe level (NSL), which has good potential for wide-angle scanning. Both simulation and experiment have been done; the experiment results show that, by applying this novel symmetric thinned array with pattern reconfigurable quasi-Yagi antenna, the scanning range of the array is $-70^{\circ} \sim 70^{\circ}$ in $H$-plane with SLL almost $-10 \mathrm{~dB}$ below the maximum of the main beam. The $3 \mathrm{~dB}$ beam-width coverage is $-86^{\circ} \sim 86^{\circ}$, which means that the proposed array can realize the entire upper-space beam coverage and restrain the SLL at the same time.
\end{abstract}

\section{Introduction}

Phased array has received abundant applications in wireless communication and radar system in recent years due to its advantages such as high reliability, rapid speed, and high accuracy of beam scanning. Particularly, microstrip antennas are good candidates for phased array application because of their low cost, ease of fabrication, and integration with microwave integrated circuits (MICs) [1]. According to the antenna theory, a microstrip phased array can scan its main beam from $-50^{\circ}$ to $50^{\circ}$ with the array gain reduction of $4-5 \mathrm{~dB}$ down from its maximum because of the limitation of radiation pattern of array element and mutual coupling between elements, which will have huge influence on the radiation pattern when the scanning angle is large $[2,3]$. It obviously restrains the further development and application of the phased array. Therefore, the improvement of scanning range of phased array and suppression of mutual coupling between elements in the array are desired.

Generally, there are two main aspects to promote the wide-angle scanning performance of phased array. One way is focused on the antenna element. A lot of research and efforts have been made to enlarge the radiation pattern of antenna element [4-7]. In [4], composite dielectric substrate is used to expand the $3 \mathrm{~dB}$ beam width of antenna element. A stable wide beam width in both $E$ and $H$ planes can be realized by using gaps and stubs-loaded patch in microstrip antenna [5]. The $3 \mathrm{~dB}$ beam width of this novel antenna in both main planes is from $-60^{\circ}$ to $60^{\circ}$. In $[6,7]$, the cavity architecture is used to reduce the mutual coupling of element in the array. The array can realize the wide-angle scanning up to $60^{\circ}$ at the operating frequency band. However, it is difficult to expand the scanning angle further just by enlarging the radiation pattern of antenna element. And wide-beam antenna element also leads to large grating lobes and side lobes when the scanning angle is large and the array is equally spaced with distance between elements greater than $0.5 \lambda$ ( $\lambda$ is the wavelength in free space).

In recent years, the existence of pattern reconfigurable antenna provides a new way to widen the radiation pattern of element. Pattern reconfigurable antenna can reconfigurate its radiation pattern by changing the state of switches integrated 
on the antenna element or feeding network, and the radiation pattern can jointly cover a wide range, which makes it a promising solution to overcome the obstacle in wide-angle scanning of the phased array $[8,9]$.

The other way is focused on the array factor. For example, a lot of optimization algorithms have been proposed, which can be used to optimize the amplitude, phase, and distance between elements of the array $[10,11]$. However, it is time consuming and sometimes cannot be used in reality because the distance between elements is too small. Some conformal arrays have been proposed to extend the scanning angle [12, 13 ], but the application of conformal array is often subject to the conditions of application environments. In $[14,15]$, the theory of unequally spaced array has been proposed, which has no grating lobes in the visible region when the distance between elements is greater than $\lambda / 2$. The mutual coupling between elements is small because the distance between adjacent elements is large, typically greater than $\lambda / 2$. The distribution of array elements can be easily calculated by formula.

So the combination of pattern reconfigurable antenna and thinned array will have much potential in the ability of wideangle scanning of antenna array. In [16], applying weighted thinned array with pattern reconfigurable antenna has been done; the proposed array can scan its main beam in the range of $-60^{\circ} \sim 60^{\circ}$ in $\mathrm{H}$-plane.

In this paper, a novel antenna thinned array with symmetric distribution along the array center is proposed. The pattern of the proposed array can be properly adjusted by changing the weighted function and the amplitude of the weighted factor, which releases more degrees of freedom in array design. By properly adjusting the weighted function and weighted factor, the SLL can be suppressed. By applying this novel symmetric thinned array with pattern reconfigurable quasi-Yagi antenna, the scanning range of the array is $-70^{\circ} \sim$ $70^{\circ}$ in $H$-plane with SLL almost $-10 \mathrm{~dB}$ below the maximum of the main beam. The $3 \mathrm{~dB}$ beam-width coverage is $-86^{\circ} \sim$ $86^{\circ}$. The simulation results agree well with the experiment results, which shows the validation of the proposed method in wide-angle scanning array design.

\section{Thinned Array Design}

Traditionally, the distribution of elements in phased array is equally spaced and the distance between elements is smaller than $\lambda / 2$ to avoid grating lobes in wide-angle scanning. However, small distance will cause strong mutual coupling between elements, which has huge influence on the radiation pattern and reflection coefficient of elements, and sometimes it might cause the scanning blindness. Besides, the size of some antenna elements is equal to or bigger than $\lambda / 2$, so some solutions should be figured out to use these elements in the array. Applying weighted thinned array is a good choice to overcome this difficulty [16]. Not only can the distance and amplitude of antenna elements be adjusted, but the grating lobes can be eliminated at the same time.

The radiation pattern of $N$ isotropic elements can be expressed as

$$
E(\theta)=\sum_{n=1}^{N} I_{n} e^{j k s_{n} \sin \theta}
$$

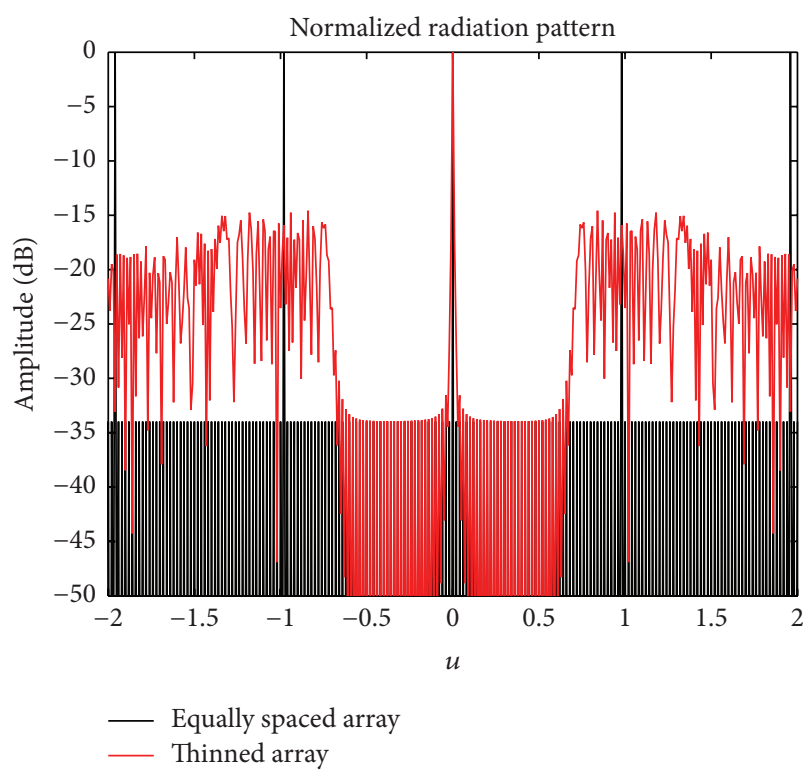

FIGURE 1: Radiation pattern of equally spaced and thinned array.

where $I_{n}$ stands for the current of $n$th element, $k=2 \pi / \lambda$ is the wave number, $s_{n}$ stands for the distance of $n$th element to the reference point, and $\theta$ is the angle of the radiation pattern. The formula can be further simplified as

$$
E(\mu)=\sum_{n=1}^{N} w_{n} e^{j k s_{n} \mu}
$$

where $w_{n}$ is the current amplitude of $n$th element and $\mu=$ $\sin \theta-\sin \theta_{0}$. This formula can be rewritten as a summation of series of grating lobes and one main lobe [14]:

$$
\begin{aligned}
& E(\mu)=\sum_{m=-\infty}^{\infty}(-1)^{m(N-1)} E_{m}(\mu) \\
& E_{m}(\mu) \\
& \quad=\frac{1}{2} \int_{-1}^{1} w(x) \frac{d y}{d x} e^{-j \psi(x)+j m \pi N(y-x)} e^{j(\mu+m \pi N) x} d x .
\end{aligned}
$$

In (3), $E_{m}(\mu)$ stands for the $m$ th grating lobes of the array. The grating lobes can be effectively avoided by exponentially spacing the elements of the array [15]. Figure 1 shows the radiation pattern of 50 isotropic elements which are equally spaced and exponentially spaced. The length of the array is $50 \lambda$. It can be seen clearly from the picture that the grating lobes have been inhibited by using exponentially thinned array in the visible region.

The radiation pattern of exponentially thinned array can be adjusted by changing the ratio of the maximum distance to minimum distance. And the amplitude of current of array element can also be adjusted by introducing a new weighted function [17]. In [17], Zhang proposed a general raised cosine function to weight the exponentially thinned array. The side lobe level near the main beam (NSL) can be reduced further. 
However, the peak side lobe lever (PSL) is still high when the number of elements of the array is small. So it might not be the good choice for the phased array to realize the wideangle scanning, especially for the array with small number of elements, which might not achieve the optimal solution. And the mutual coupling should also be considered while selecting the weighted function and weighted factor.

In order to enlarge the scanning angle and suppress the SLL at the same time, a compromise should be made between PSL and NSL of the array factor. In addition, the weighted function and factor should be carefully selected and optimized. Much simulation has been done in order to find the desired weighted function to reduce the SLL in wide-angle scanning. The weighted function can be expressed as

$$
w(v)=a+(1-a)\left[1-\sin \frac{\pi(v-1)}{2(N-1)}\right]
$$

where $N$ stands for the total number of elements in the array and $\mathbf{a}$ is the weighted factor from 0 to 0.5 . By placing the array symmetrically, the SLL can be further reduced. The position of each element can be calculated by the following:

$$
\begin{aligned}
X_{v} & =\frac{L}{2 \int_{1}^{(N+1) / 2} r^{h(i)} d i} \int_{1}^{v} r^{h(i)} d i \\
h(v) & =\frac{\int_{1}^{v}|w(i)|^{2} d i}{\int_{1}^{(N+1) / 2}|w(i)|^{2} d i} .
\end{aligned}
$$

$X_{v}$ is the distance of $v$ th elements to the reference point 0 and $\mathbf{r}$ can be properly adjusted in the range of 1.5 to 2.5 , which represents the ratio of maximum distance to minimum distance between elements. Once the length of the array is determined, wide-angle scanning performance can be realized by optimizing the parameters such as $\mathbf{r}$, array factor, and the number of the elements.

\section{Pattern Reconfigurable Quasi-Yagi Antenna}

The pattern reconfigurable antenna element used in this paper is quasi-Yagi pattern reconfigurable antenna [16]. The geometry of the antenna mentioned above is shown in Figure 2. The antenna is printed on the Rogers 5880 substrate with permittivity of 2.2 , height of $8 \mathrm{~mm}$, and operating frequency of $5.8 \mathrm{GHz}$. It consists of three parallel metal strips used as radiators and a DC bias circuit. The driven strip is in the middle of two parasitic strips. The length of the excitation strip is about $0.5 \lambda_{g}$ ( $\lambda_{g}$ is the guided wavelength in operating frequency), which is a little shorter than two parasitic strips. The distance between strips is about $0.25 \lambda$. The antenna is fed by a SMA connector with its inner conductor soldered to the driven strip and outer conductor soldered to the ground plane. Six inductances, that is, $L_{1} \sim L_{6}$, are inserted in two parasitic strips to block the RF signal to the DC circuit. High impedance lines connected with shorting pins are used to form the DC circuit and reduce the impact on radiation pattern by DC circuit. The parameters for the pattern reconfigurable antenna are given in Table 1.
TABLE 1: Parameter for pattern reconfigurable antenna.

\begin{tabular}{lc}
\hline Parameters & Value $(\mathrm{mm})$ \\
\hline$L_{g}$ & 70 \\
$w_{g}$ & 60 \\
$w$ & 1.5 \\
$g$ & 6 \\
$L_{m}$ & 20 \\
$L_{r}$ & 22 \\
$d_{l}$ & 3.5 \\
$s$ & 10 \\
\hline
\end{tabular}

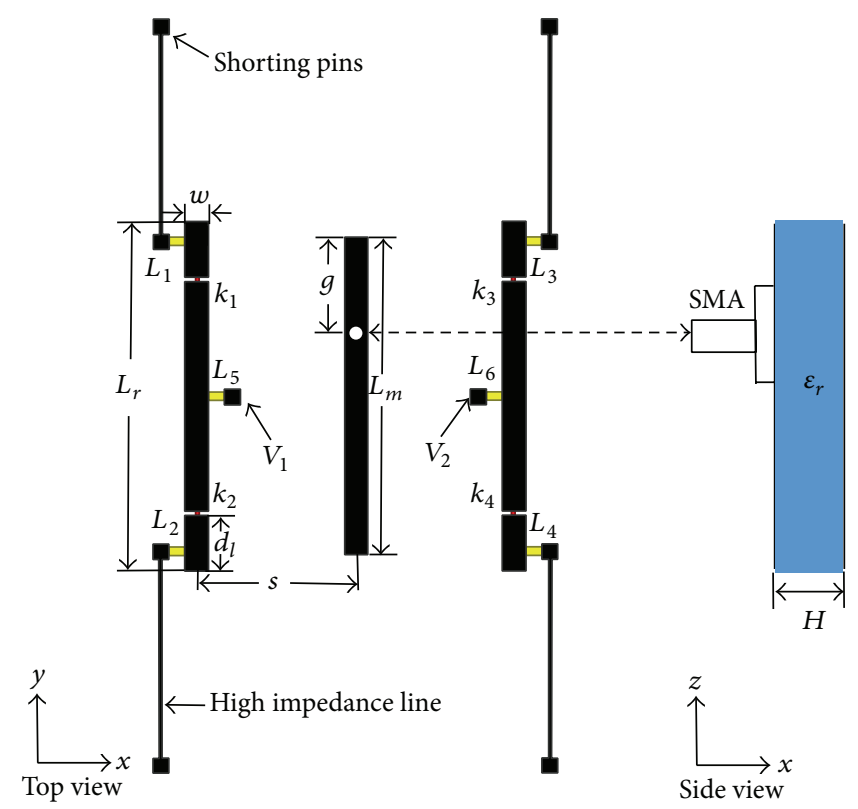

FIGURE 2: Geometry of the pattern reconfigurable antenna.

Four PIN diodes integrated on the two parasitic strips are used as switches to change the radiation pattern of the antenna just by transforming the state of the switches to be "ON" or "OFF." The proposed antenna can operate in three modes, that is, L-mode, B-mode, and R-mode. Lmode stands for the radiation pattern of the pattern reconfigurable antenna tilting to the left side. B-mode stands for the radiation pattern of the pattern reconfigurable antenna tilting to the broad side, and R-mode stands for the radiation pattern of the pattern reconfigurable antenna tilting to the right side.

When the voltage of $-5 \mathrm{~V}$ is applied in the point $V_{1}$ and $5 \mathrm{~V}$ is applied in the point $V_{2}$, the PIN diodes in the left parasitic strip are in "OFF" state while the PIN diodes in the right parasitic strip are in "ON" state, and the antenna operates in L-mode. When both points apply the voltage of $-5 \mathrm{~V}$, the antenna is in B-mode. When the voltage of $5 \mathrm{~V}$ is applied in the point $V_{1}$ and $-5 \mathrm{~V}$ is applied in the point $V_{2}$, the antenna is in R-mode. The PIN diode used in simulation is MA4GP907 with equivalent resistance of $4 \Omega$ and capacitance 

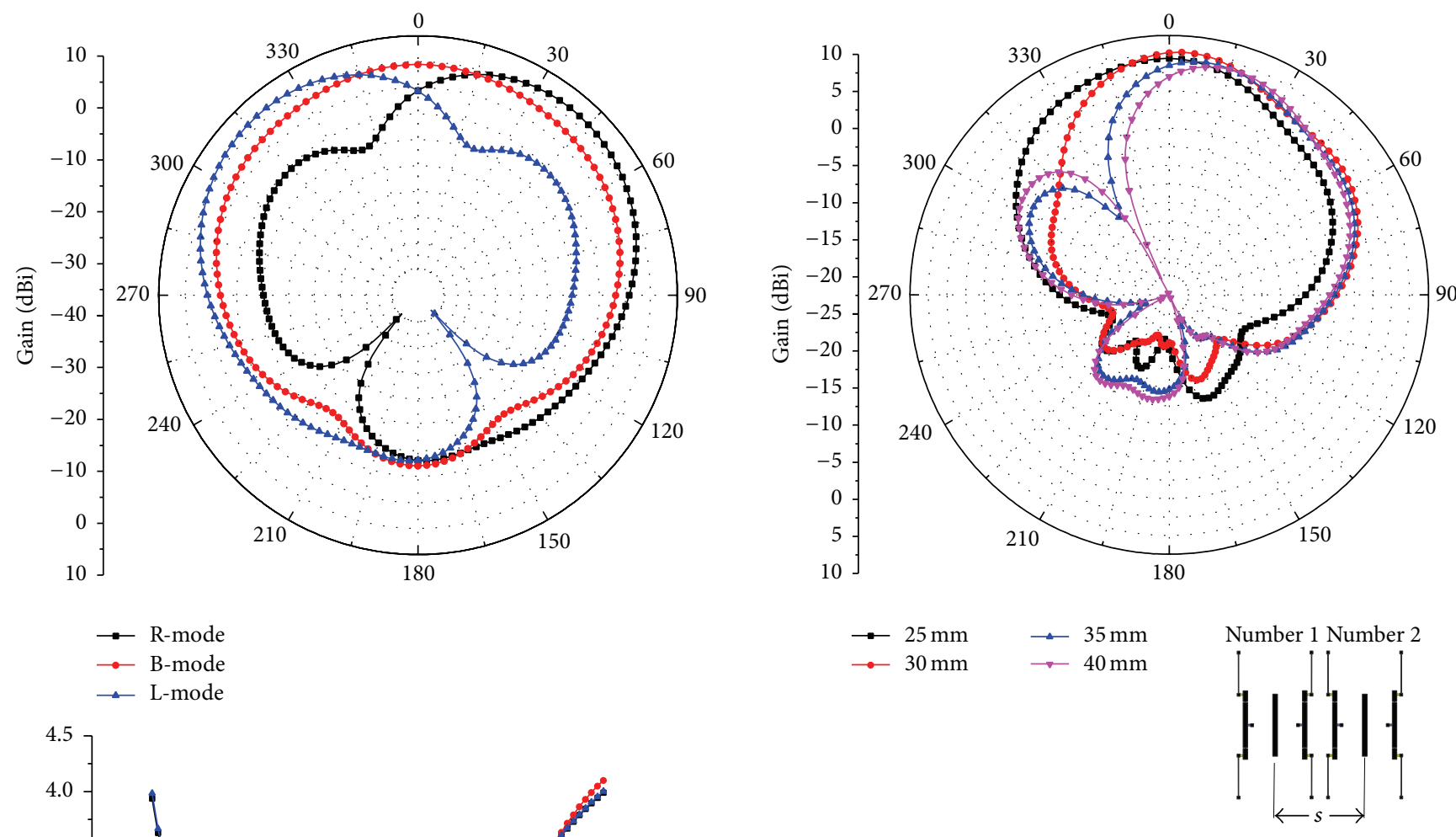

Figure 4: Parameter study of the distance between two elements.

the distance becomes large, because the mutual coupling of the two elements becomes small when the distance is large and strong when the distance is small. It indicates the usefulness of thinned array in wide-angle scanning performance because the distance between elements is generally larger than $0.5 \lambda$ and the grating lobes can be eliminated at the same time.

\section{Analysis of the Experimental Results}

FIGURE 3: Radiation pattern and VSWR of the proposed antenna.

of $0.025 \mathrm{pf}$. The simulation results can be found in Figure 3 . The $3 \mathrm{~dB}$ beam width of the antenna can jointly cover an angle in the range of $-75^{\circ} \sim 75^{\circ}$; all of the three modes share a common $2: 1$ VSWR bandwidth from $5.68 \mathrm{GHz}$ to $6 \mathrm{GHz}$.

It is worth mentioning that the tilting angle of the single quasi-Yagi reconfigurable antenna in R-mode and L-mode might have much difference in the array because of the mutual coupling between elements. Figure 4 shows the radiation pattern of different distance between elements of antenna element number 1 in R-mode in the existence of another element number 2 that is connected with the matched load of $50 \Omega$. It can be found that the radiation pattern of number 1 nearly directs to broad side when the distance between two elements is small and gradually tilts to the right side when

In order to testify the ability of the symmetric weighted thinned array proposed in Section 2 in wide-angle scanning, a 7-element symmetric thinned array has been designed and fabricated. The photograph of the proposed array can be found in Figure 5. The total length of the array is $5 \lambda, \mathbf{r}=2$, and the weighted factor is chosen to be 0.5 . The distribution of elements can be calculated by (5), and the amplitude of elements can be figured out by (4). The position and amplitude of elements are shown in Table 2. The element used in the array is the quasi-Yagi reconfigurable antenna. And only L-mode and R-mode are used. When the array scans its main beam to the right side, all the elements operate in $\mathrm{R}$ mode. When the array scans its main beam to the left side, all the elements operate in L-mode. When the array scans its main beam to the broad side, both L-mode and R-mode can be used.

The scattering matrix $s_{m n}(m, n=1,2,3, \ldots, 7)$ of the prototype array is measured using an Agilent E8361A Vector Network Analyzer. During the measurement of $S$-parameter 
TABLE 2: Position and amplitude of the elements.

\begin{tabular}{lcccc}
\hline & Number 1 & Number 2 & Number 3 & Number 4 \\
\hline$w$ & 1 & 0.75 & 0.567 & 0.5 \\
$X(\mathrm{~mm})$ & 0 & 30.27 & 69.94 & 116.38 \\
\hline & & Number 5 & Number 6 & Number 7 \\
\hline$w$ & 0.75 & 0.567 & 0.5 \\
$X(\mathrm{~mm})$ & & -30.27 & -69.94 & -116.38 \\
\hline
\end{tabular}

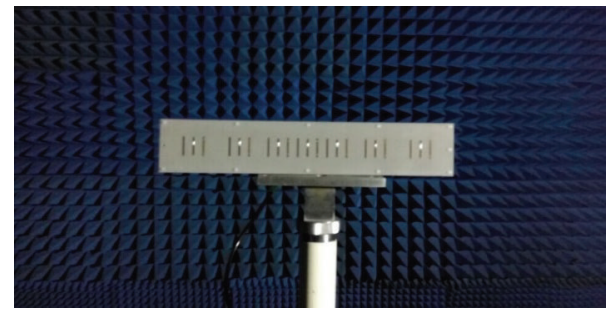

(a)

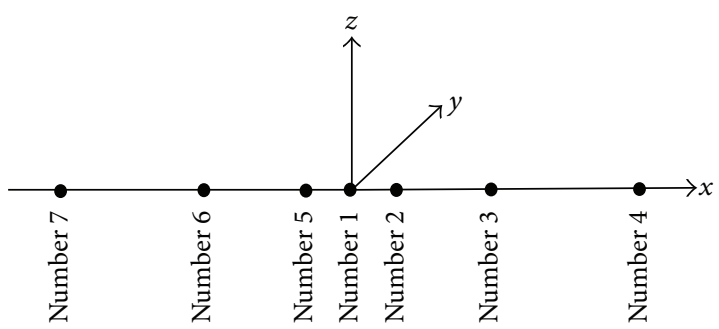

(b)

FIGURE 5: Configuration and distribution of the symmetric weighted thinned array. (a) Prototype of the array. (b) Distribution.

of each element in the array, all the remaining elements are connected with the matched load. The measured scattering matrix is used to compute the active reflection coefficients by (7) [18], where $\theta$ is the scanning angle of the array and $d_{m}$ is the distance from $m$ th element to the reference plane:

$$
\Gamma_{m}(\theta)=e^{j k d_{m} \sin (\theta)} \sum_{n=1}^{7} s_{m n} e^{-j k d_{n} \sin (\theta)} .
$$

The measured active reflection coefficients at the operating frequency are depicted in Figure 6. As can be seen from Figure 6, good impedance matching is achieved over the whole beam scanning range.

Because of the symmetry of the array, only R-mode is measured in the experiment to scan from broadside to the right side, and the performance of array scanning in the left side will be the same. The results of the scanning performance in both simulation and experiment are shown in Figure 7; the simulation result agrees well with the experiment result, which validates the effectiveness of the proposed symmetric weighted thinned array in the performances of wide-angle scanning and SLL reduction. The measurement results show that the $3 \mathrm{~dB}$ beam-width coverage is $-9^{\circ} \sim 86^{\circ}$ when all elements are operating in R-mode. So the beam width of the proposed array can jointly cover the entire upper space.
TABLE 3: Simulated scanning characteristics of the prototype.

\begin{tabular}{lccc}
\hline$\theta$ & Gain $(\mathrm{dBi})$ & $3 \mathrm{~dB}$ beam-width coverage & SLL $(\mathrm{dB})$ \\
\hline $0^{\circ}$ & 10.66 & $-7^{\circ} \sim 9^{\circ}$ & 11.1 \\
$10^{\circ}$ & 12.19 & $4^{\circ} \sim 16^{\circ}$ & 11.4 \\
$20^{\circ}$ & 12.23 & $14^{\circ} \sim 27^{\circ}$ & 11.9 \\
$30^{\circ}$ & 11.99 & $23^{\circ} \sim 36^{\circ}$ & 12.0 \\
$40^{\circ}$ & 11.43 & $34^{\circ} \sim 47^{\circ}$ & 10.7 \\
$50^{\circ}$ & 10.04 & $42^{\circ} \sim 57^{\circ}$ & 9.0 \\
$60^{\circ}$ & 8.49 & $50^{\circ} \sim 71^{\circ}$ & 8.9 \\
$70^{\circ}$ & 9.01 & $59^{\circ} \sim 86^{\circ}$ & 10.5 \\
$75^{\circ}$ & 9.05 & $64^{\circ} \sim 96^{\circ}$ & 10.7 \\
\hline
\end{tabular}

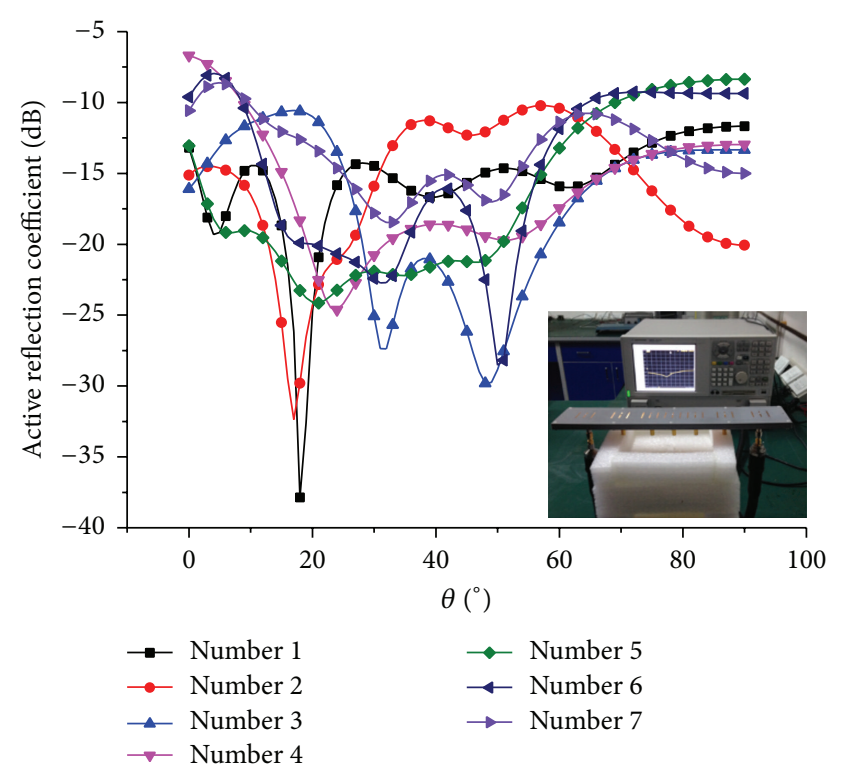

FIGURE 6: Measured active reflection coefficients of the sevenelement phased array.

The maximum gain and SLL of the proposed array when scanning from broad side to the end-fire direction are shown in Figure 8. More details can be found in Tables 3 and 4 . As can be seen, the thinned array can scan its main beam in the range of $0^{\circ} \sim 70^{\circ}$ with the SLL almost $-10 \mathrm{~dB}$ below the maximum of the main beam except for the scanning angles $60^{\circ}(-8.2 \mathrm{~dB})$ and $70^{\circ}(-7.3 \mathrm{~dB})$. The gain fluctuation is almost less than $3 \mathrm{~dB}$ except for the scanning angles $60^{\circ}$ $(4 \mathrm{~dB})$ and $70^{\circ}(3.27 \mathrm{~dB})$. In general, the simulation results agree well with the experiment results, which indicates the validation of the novel symmetric weighted thinned array in the performance of wide-angle scanning with low SLL.

\section{Conclusion}

A novel symmetric weighted thinned array with pattern reconfigurable antenna as elements has been proposed. And a prototype has been fabricated. Both the simulation and measurement results show that this novel array can scan its main beam from $-70^{\circ}$ to $70^{\circ}$ in $H$-plane with SLL almost 


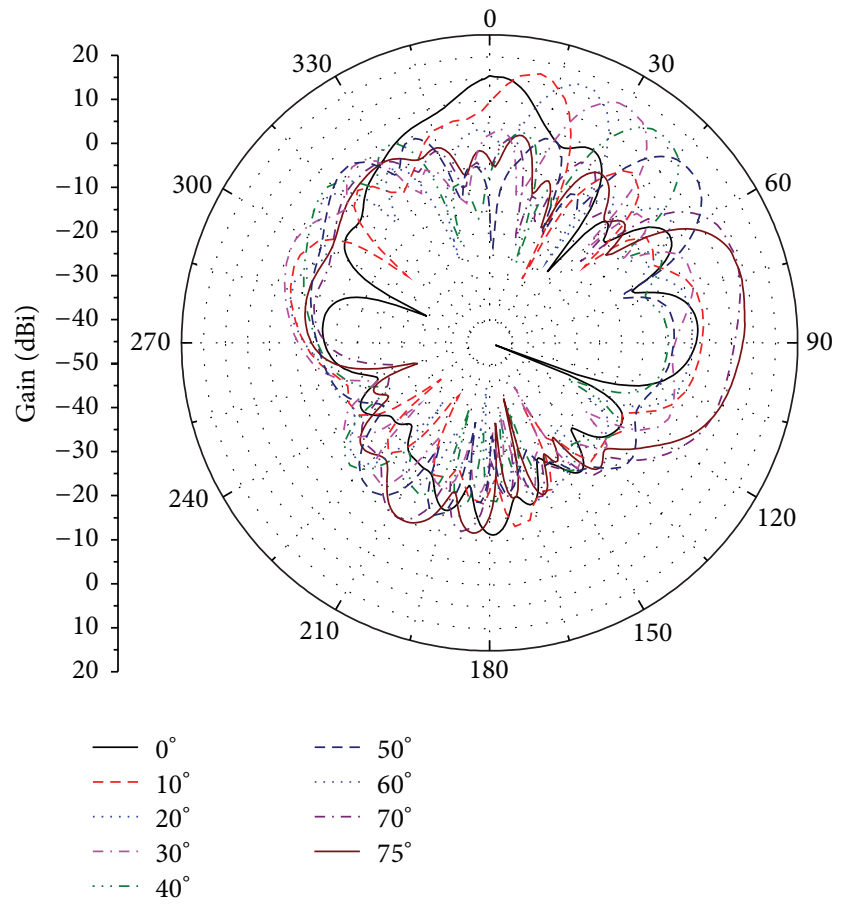

(a)

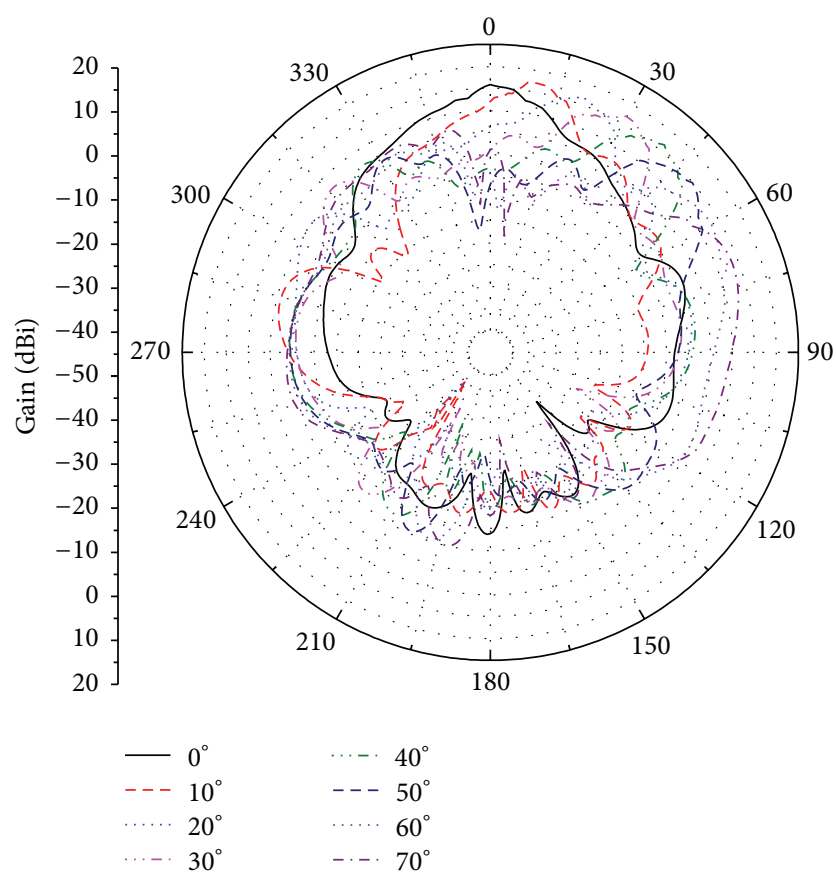

(b)

FIGURE 7: Scanning performance of the symmetric weighted thinned array. (a) Simulated results. (b) Measured results.

$-10 \mathrm{~dB}$ below the maximum of the main beam. The $3 \mathrm{~dB}$ beam-width coverage is $-86^{\circ} \sim 86^{\circ}$, which means that this novel symmetric thinned antenna array can realize the entire upper-space beam coverage and restrain the SLL at the same time.
TABLE 4: Measured scanning characteristics of the prototype.

\begin{tabular}{lccc}
\hline$\theta$ & Gain $(\mathrm{dBi})$ & $3 \mathrm{~dB}$ beam-width coverage & SLL $(\mathrm{dB})$ \\
\hline $0^{\circ}$ & 10.78 & $-8^{\circ} \sim 8^{\circ}$ & 14.29 \\
$10^{\circ}$ & 12.02 & $5^{\circ} \sim 17^{\circ}$ & 10.38 \\
$20^{\circ}$ & 11.85 & $14^{\circ} \sim 27^{\circ}$ & 11.46 \\
$30^{\circ}$ & 11.80 & $24^{\circ} \sim 37^{\circ}$ & 10.08 \\
$40^{\circ}$ & 11.52 & $33^{\circ} \sim 46^{\circ}$ & 10.1 \\
$50^{\circ}$ & 9.95 & $41^{\circ} \sim 56^{\circ}$ & 10.03 \\
$60^{\circ}$ & 8.02 & $49^{\circ} \sim 72^{\circ}$ & 8.21 \\
$70^{\circ}$ & 8.75 & $60^{\circ} \sim 86^{\circ}$ & 7.36 \\
\hline
\end{tabular}

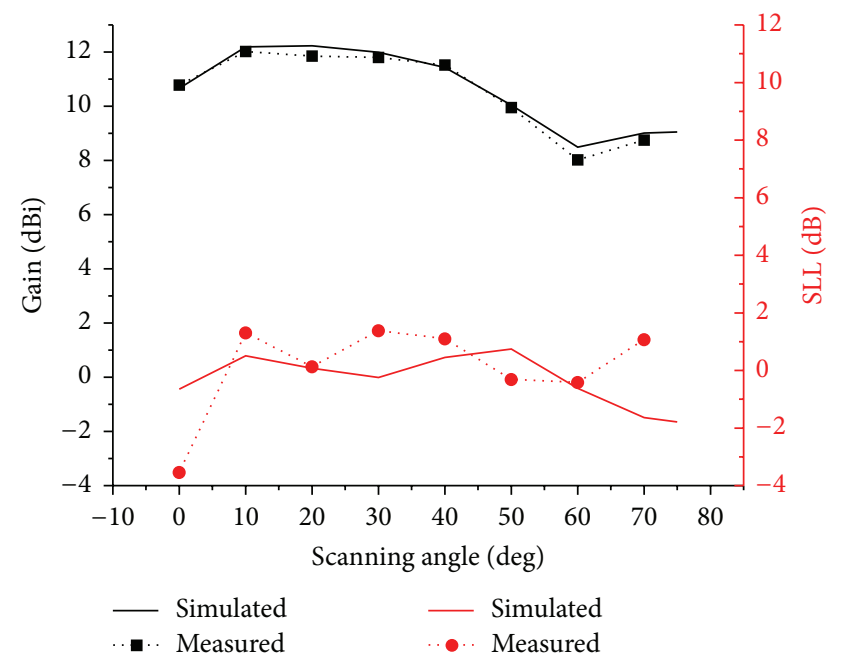

FIGURE 8: Maximum gain and SLL when the array scans its main beam from $0^{\circ}$ to $70^{\circ}$.

\section{Competing Interests}

The authors declare that there are no competing interests regarding the publication of this paper.

\section{Acknowledgments}

This work has been supported by the Advanced Research Foundation under Grant nos. 9140A07030514DZ02101 and 9140A07010715DZ02001.

\section{References}

[1] F. W. Schmidt, "Low-cost microstrip phased array antenna for use in mobile satellite telephone communication service," in Proceedings of the IEEE Antennas and Propagation Society International Symposium, vol. 25, pp. 1152-1155, Blacksburg, VA, USA, June 1987.

[2] R. J. Mailloux, Phased Array Antenna Handbook, Artech House, Norwood, Mass, USA, 2nd edition, 2008.

[3] C. A. Balanis, Antenna Theory, Analysis, and Design, John Wiley \& Sons, Hoboken, NJ, USA, 2nd edition, 1997.

[4] S. Chattopadhyay, J. Y. Siddiqui, and D. Guha, "Rectangular microstrip patch on a composite dielectric substrate for high-gainwide-beam radiation patterns," IEEE Transactions on Antennas and Propagation, vol. 57, no. 10, pp. 3325-3328, 2009. 
[5] Z.-S. Duan, S.-B. Qu, Y. Wu, and J.-Q. Zhang, "Wide bandwidth and broad beamwidth microstrip patch antenna," Electronics Letters, vol. 45, no. 5, pp. 249-250, 2009.

[6] S. E. Valavan, D. Tran, A. G. Yarovoy, and A. G. Roederer, "Planar dual-band wide-scan phased array in X-band," IEEE Transactions on Antennas and Propagation, vol. 62, no. 10, pp. 5370-5375, 2014.

[7] S. E. Valavan, D. Tran, A. G. Yarovoy, and A. G. Roederer, "Dualband wide-angle scanning planar phased array in X/Ku-Bands," IEEE Transactions on Antennas and Propagation, vol. 62, no. 5, pp. 2514-2521, 2014.

[8] X.-S. Yang, B.-Z. Wang, S. H. Yeung, Q. Xue, and K. F. Man, "Circularly polarized reconfigurable crossed-yagi patch antenna," IEEE Antennas and Propagation Magazine, vol. 53, no. 5, pp. 65-80, 2011.

[9] X. Ding, B.-Z. Wang, and G.-Q. He, "Research on a millimeterwave phased array with wide-angle scanning performance," IEEE Transactions on Antennas and Propagation, vol. 61, no. 10, pp. 5319-5324, 2013.

[10] P. J. Bevelacqua and C. A. Balanis, "Minimum sidelobe levels for linear arrays," IEEE Transactions on Antennas and Propagation, vol. 55, no. 12, pp. 3442-3449, 2007.

[11] M. G. Bray, D. H. Werner, D. W. Boeringer, and D. W. Machuga, "Optimization of thinned aperiodic linear phased arrays using genetic algorithms to reduce grating lobes during scanning," IEEE Transactions on Antennas and Propagation, vol. 50, no. 12, pp. 1732-1742, 2002.

[12] Q. Wu, M. Liu, and Z.-R. Feng, "A millimeter-wave conformal phased microstrip antenna array on a cylindrical surface," in Proceedings of the IEEE Antennas and Propagation Society International Symposium (AP-S '08), vol. 1-9, pp. 1-4, IEEE, San Diego, Calif, USA, July 2008.

[13] M. Liu, Z. R. Feng, F. Y. Meng, F. L. Sun, and Q. Wu, "A $35 \mathrm{GHz}$ cone conformal microstrip $4 \times 4$ array," in Proceedings of the Asia-Pacific Microwave Conference (APMC '07), pp. 1-4, Bangkok, Thailand, December 2007.

[14] A. Ishimaru, "Theory of unequally-spaced arrays," IRE Transactions on Antennas and Propagation, vol. 10, no. 6, pp. 691-702, 1962.

[15] Y. L. Chow, "On grating plateaux of nonuniformly spaced arrays," IEEE Transactions on Antennas and Propagation, vol. 13, no. 2, pp. 208-215, 1965.

[16] Y.-Y. Bai, S. Xiao, M.-C. Tang, Z.-F. Ding, and B.-Z. Wang, "Wide-angle scanning phased array with pattern reconfigurable elements," IEEE Transactions on Antennas and Propagation, vol. 59, no. 11, pp. 4071-4076, 2011.

[17] Y. H. Zhang, "Optimumthinning of weighted linear arrays," Acta Electronica Sinica, vol. 18, no. 5, pp. 34-39, 1990.

[18] D. M. Pozar, “The active element pattern," IEEE Transactions on Antennas and Propagation, vol. 42, no. 8, pp. 1176-1178, 1994. 


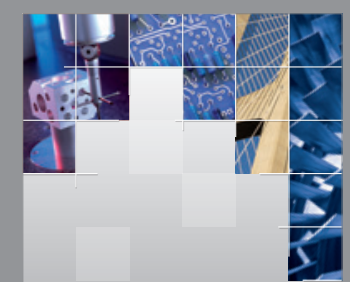

\section{Enfincering}
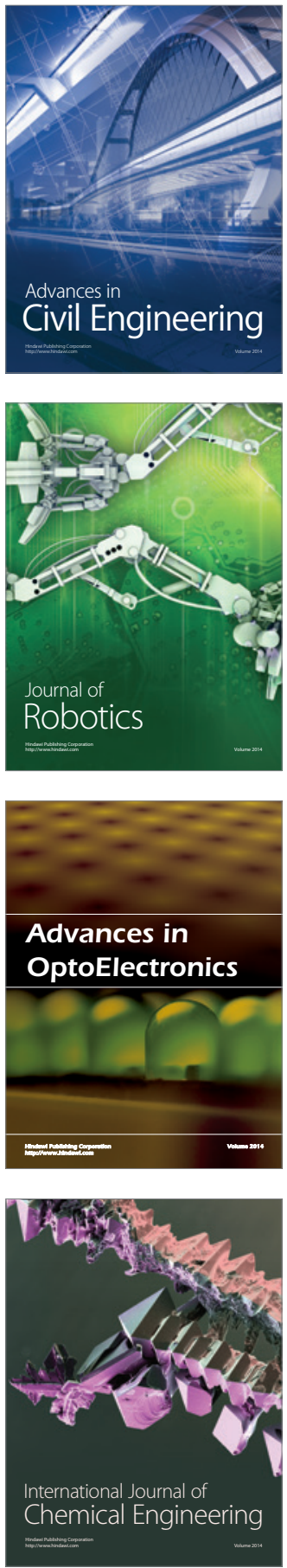

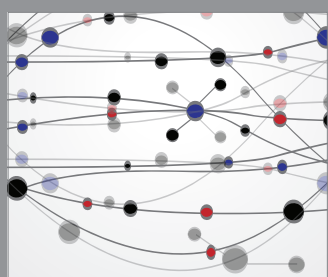

The Scientific World Journal

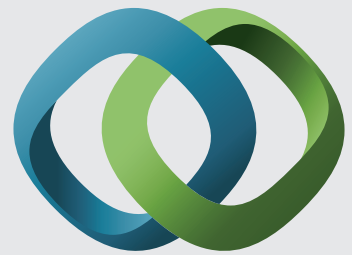

\section{Hindawi}

Submit your manuscripts at

http://www.hindawi.com
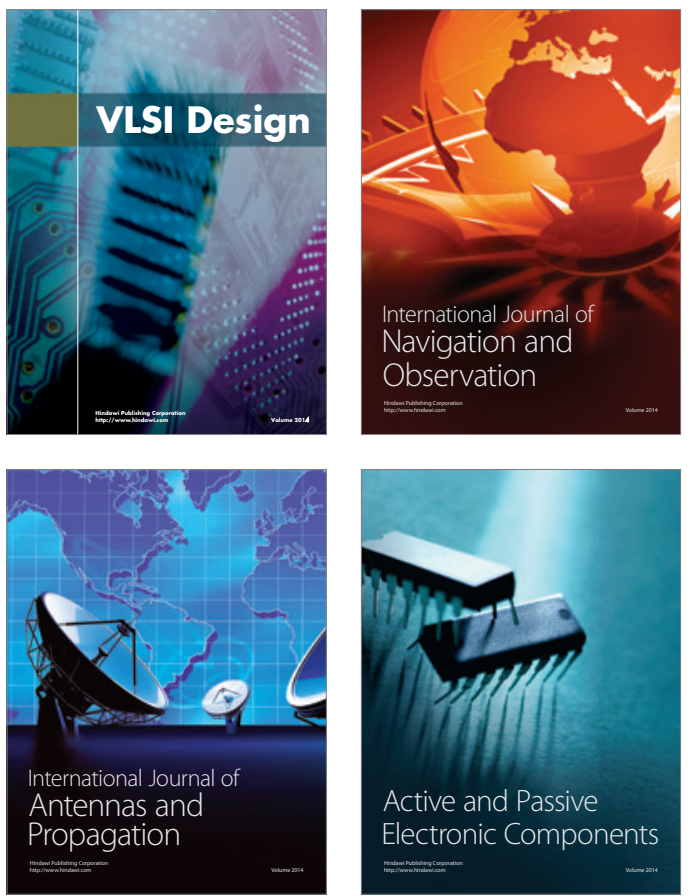
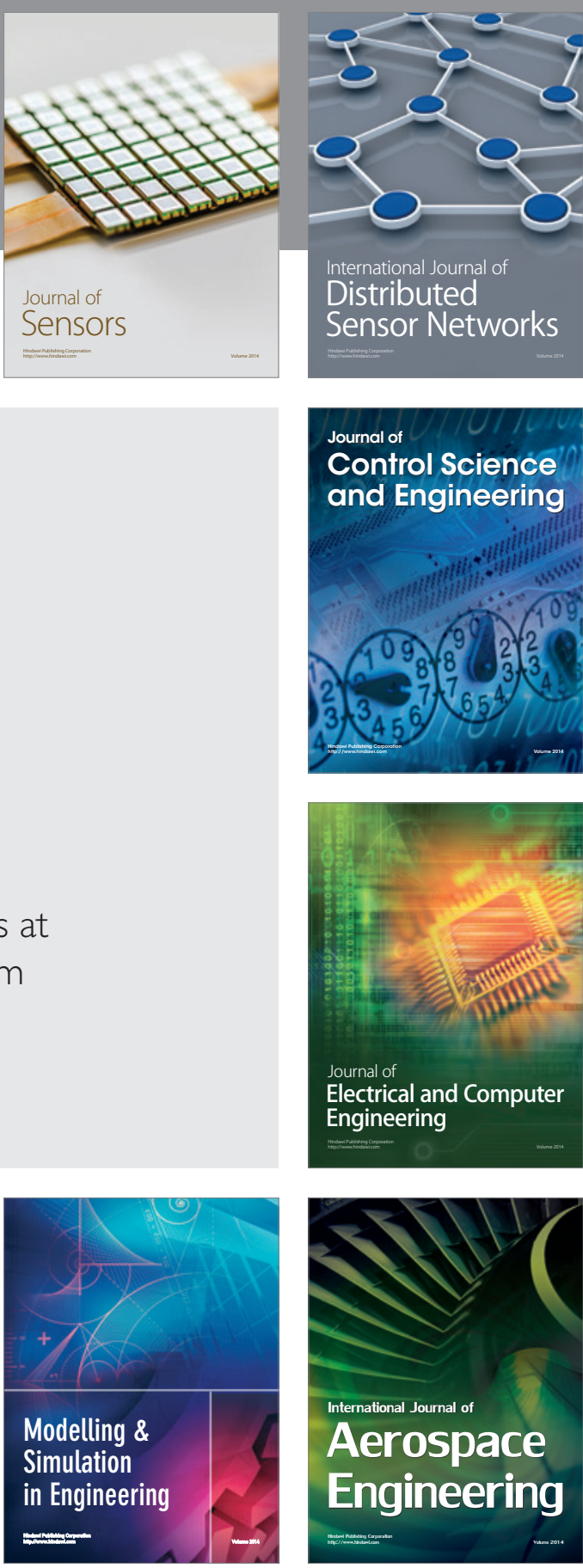

International Journal of

Distributed

Sensor Networks

Journal of

Control Science

and Engineering
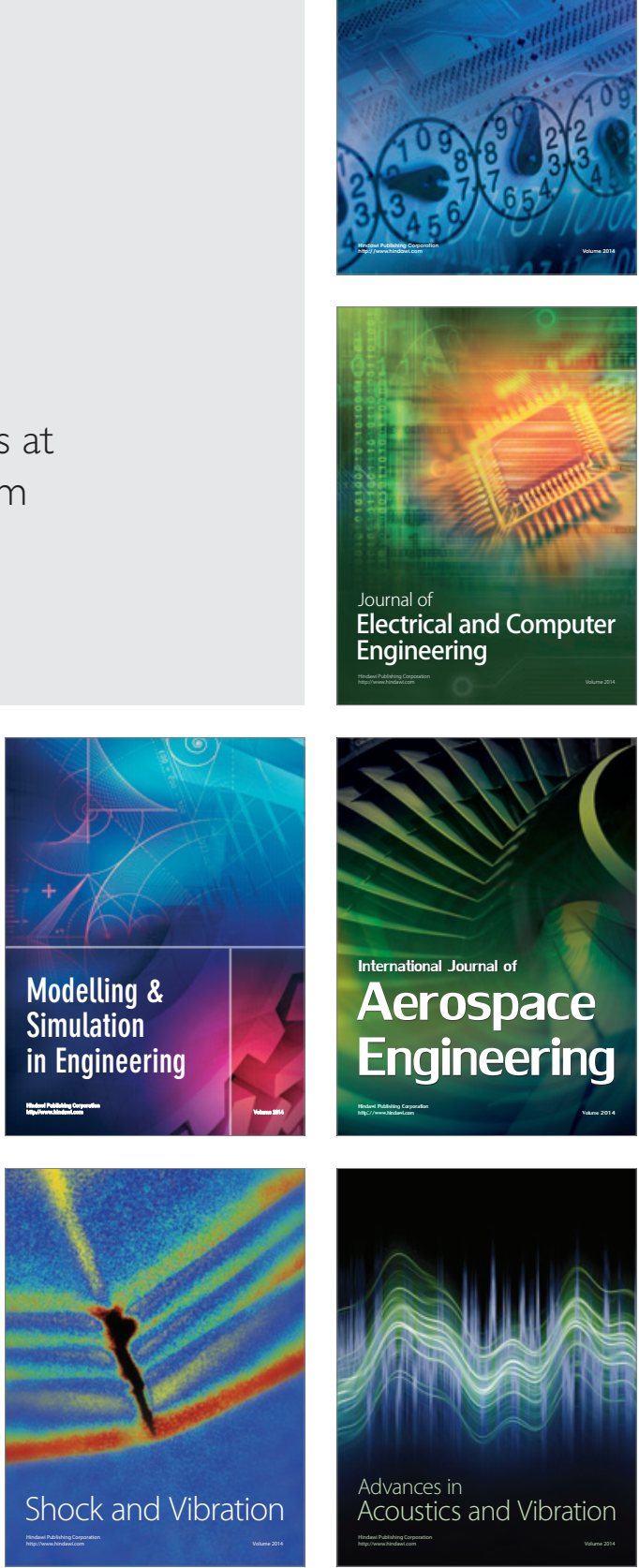